\title{
NEURAL NETWORK ADAPTIVE CONTROL OF WING-ROCK MOTION OF AIRCRAFT MODEL MOUNTED ON THREE-DEGREE-OF-FREEDOM DYNAMIC RIG IN WIND TUNNEL
}

\author{
D. I. Ignatyev ${ }^{1,2}$ \\ ${ }^{1}$ Central Aerohydrodynamic Institute (TsAGI) \\ Zhukovsky, Moscow Region, Russia \\ ${ }^{2}$ Moscow Institute of Physics and Technology (MIPT) \\ Dolgoprudny, Moscow Region, Russia
}

\begin{abstract}
High-angles-of-attack dynamics of aircraft are complicated with dangerous phenomena such as wing rock, stall, and spin. Autonomous dynamically scaled aircraft model mounted in three-degree-of-freedom (3DoF) dynamic rig is proposed for studying aircraft dynamics and prototyping of control laws in wind tunnel. Dynamics of the scaled aircraft model in $3 \mathrm{DoF}$ manoeuvre rig in wind tunnel is considered. The model limitcycle oscillations are obtained at high angles of attack. A neural network $(\mathrm{NN})$ adaptive control suppressing wing rock motion is designed. The wing rock suppression with the proposed control law is validated using nonlinear time-domain simulations.
\end{abstract}

\section{INTRODUCTION}

A dynamics of modern aircraft at high-angles-of-attack departure is complicated with a set of dangerous phenomena due to development of the high-amplitude self-induced oscillations or spin. Roll-yaw oscillations of low frequency and large amplitude arising at angles of attack in the vicinity of $30^{\circ}$ (wing-rock motion) do not only limit maneuverable performance of the aircraft but also can lead to its crash. The great emphasis has been placed on this problem for many years. Numerous studies aimed to understand the mechanism of appearance of these self-induced oscillations and to develop an adequate nonlinear aircraft aerodynamic model have been conducted. The wing-rock dynamics has been investigated both numerically and analytically $[1-8]$. The asymptotical techniques have been applied in $[4,7]$. A set of methods for wing-rock control have been

(C) The Authors, published by EDP Sciences. This is an open access article distributed under the terms of the Creative Commons Attribution License 4.0 (http://creativecommons.org/licenses/by/4.0/). 
proposed, namely, direct adaptive control [5], nonlinear $H_{\infty}$-method [6], NN adaptive control $[5,8]$, and others. In $[5,8]$, the wing-rock motion has been considered as a one-dimensional problem, namely, self-induced roll oscillations of the slender delta wing. In the practical application for modern aircraft, self-induced oscillations at high angles of attack are much more complicated.

A technique for experimental investigation and control of critical flight regimes using dynamically scaled wind tunnel models equipped with onboard microcontroller and inertial measurement unit mounted in a $3 \mathrm{DoF}$ manoeuvre rig is developed at TsAGI. The technique is designed for identification of unsteady aerodynamic characteristics, for investigation of aircraft dynamics at high angles of attack, and for clearance of control laws at the motions that are close to the controllable motions of the aircraft in the flight conditions. As it was shown in the recent studies [9], the phenomena of nonlinear dynamics of aircraft at high angles of attack such as lateral self-induced large-amplitude oscillations can be modeled in the wind tunnel using the dynamically scaled aircraft model. These results give the possibility to investigate complex nonlinear phenomena of flight dynamics and control techniques using the dynamically scaled model in wind tunnel.

A control of the lateral self-oscillations of aircraft model in 3DoF gimbals in wind tunnel designed using LPV (Linear Parameter Varying) synthesis was proposed in [10]. At the same time, the control design for high angles of attack is complicated with the fact that it is difficult to obtain a correct mathematical model of aerodynamic characteristics of aircraft. Neural networks have been recently shown to be an effective tool to catch the complex behavior of aerodynamics at high angles of attack $[11,12]$ and to control the aircraft at presence of large uncertainties $[5,8]$. Such an active introduction of NNs is mainly connected with their universal approximation properties [13], which enable the neural networks to be used for an arbitrary aircraft without significant simplifying assumptions. The aim of this study is to design a control law using radial basis function (RBF) $\mathrm{NN}$ that stabilize the wind tunnel aircraft model in the $3 \mathrm{DoF}$ manoeuvre rig.

The paper is organized in the following way. After the introduction, a brief description of the dynamic rig is given in section 2 . In the same section the examples of aircraft dynamics at moderate and high angles of attack are presented. In section 3 , the design of the $\mathrm{NN}$ adaptive control is introduced. In section 4 , the simulation of the aircraft model behavior with adaptive control is given. Concluding remarks are given in section 5 .

\section{THREE-DEGREE-OF-FREEDOM DYNAMICS}

A schematic view of a dynamic rig in the wind tunnel is shown in Fig. 1. The sting is located along the velocity of the flow, $3 \mathrm{DoF}$ gimbals are used to achieve 


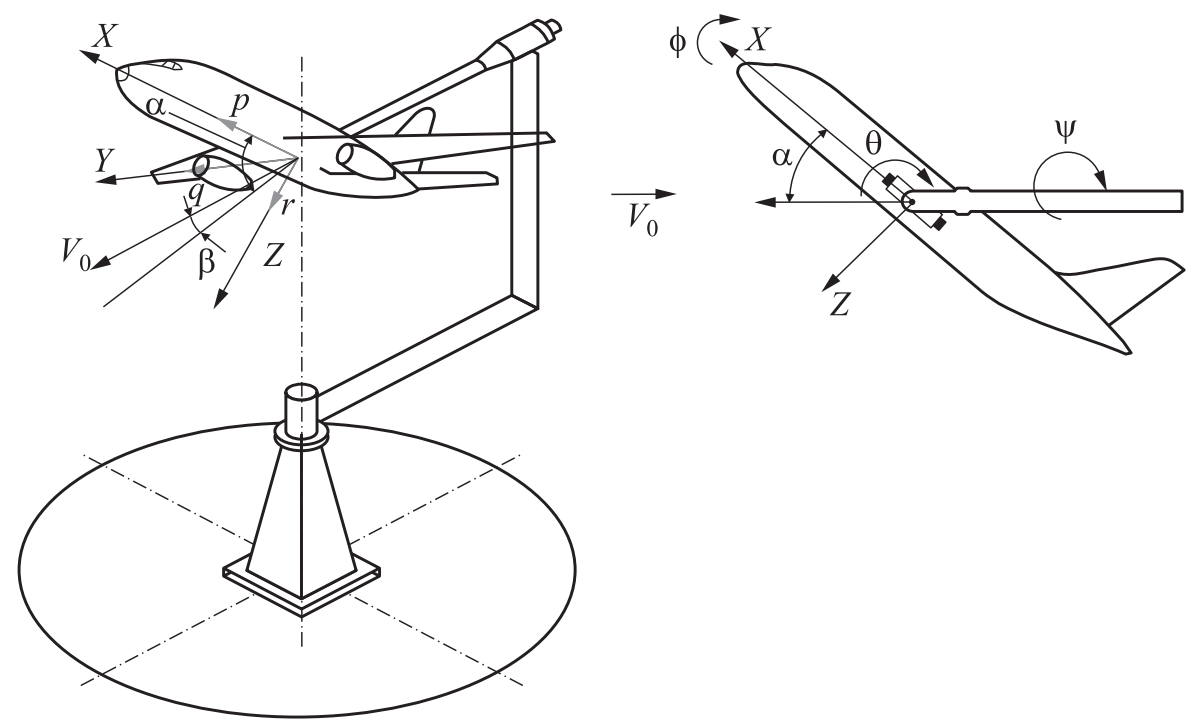

Figure 1 The dynamic rig for dynamics modeling and control clearance

the rotation in $3 \mathrm{DoF}$ : pitch, roll, and yaw. The dynamic rig is designed to be installed in the open working section of the TsAGI wind tunnel T-103 of low subsonic velocities.

The wing span of the dynamically scaled model is about $1 \mathrm{~m}$ and the mass is about $4.5 \mathrm{~kg}$. It has a conventional set of control surfaces driven by servos: differential stabilator, ailerons, and a rudder. Position of the aircraft model in the $3 \mathrm{DoF}$ gimbals is defined by three rotational angles: $\psi, \theta$, and $\phi$ (see Fig. 1). The dorsal holder allows free rotation in yaw $\psi$, pitch angle $\theta$ can be varied inside the interval $\left[20^{\circ}, 120^{\circ}\right]$, and roll angle $\phi$ lies in the range $\left[-40^{\circ}, 40^{\circ}\right]$.

The dynamics of the aircraft model in $3 \mathrm{DoF}$ gimbals is described with the following system of differential equations [9]:

$$
\left.\begin{array}{l}
\dot{\theta}=-r \sin \phi+q \cos \phi ; \\
\dot{\psi}=\frac{r \cos \phi+q \sin \phi}{\sin \theta} ; \\
\phi=p-\frac{r \cos \phi+q \sin \phi}{\operatorname{tg} \theta} ; \\
\dot{\omega}=\mathbf{J}^{-1}(-\omega \times \mathbf{J} \omega+\mathbf{M}(\alpha, \beta, \omega, \mathbf{u})) .
\end{array}\right\}
$$


Here, $\omega=(p, q, r)^{T}$ is the vector of angular velocity in the body reference system $X, Y, Z$ given in Fig. $1 ; \mathbf{u}=\left(\delta_{e}, \delta_{\Delta e}, \delta_{a}, \delta_{r}\right)^{\mathrm{T}}$ is the vector of control deflections, namely, $\delta_{e}=\left(\delta_{e_{r}}+\delta_{e_{l}}\right) / 2$ is the mean deflection of two horizontal stabilators (elevator) and $\delta_{\Delta e}=\left(\delta_{e_{r}}-\delta_{e_{l}}\right) / 2$ is the differential deflection of two horizontal stabilators, $\delta_{a}$ is the deflection of ailerons, and $\delta_{r}$ is the deflection of rudders; $\mathbf{J}$ is the model inertia tensor; and $\mathbf{M}$ is the vector of the aerodynamic moments (the
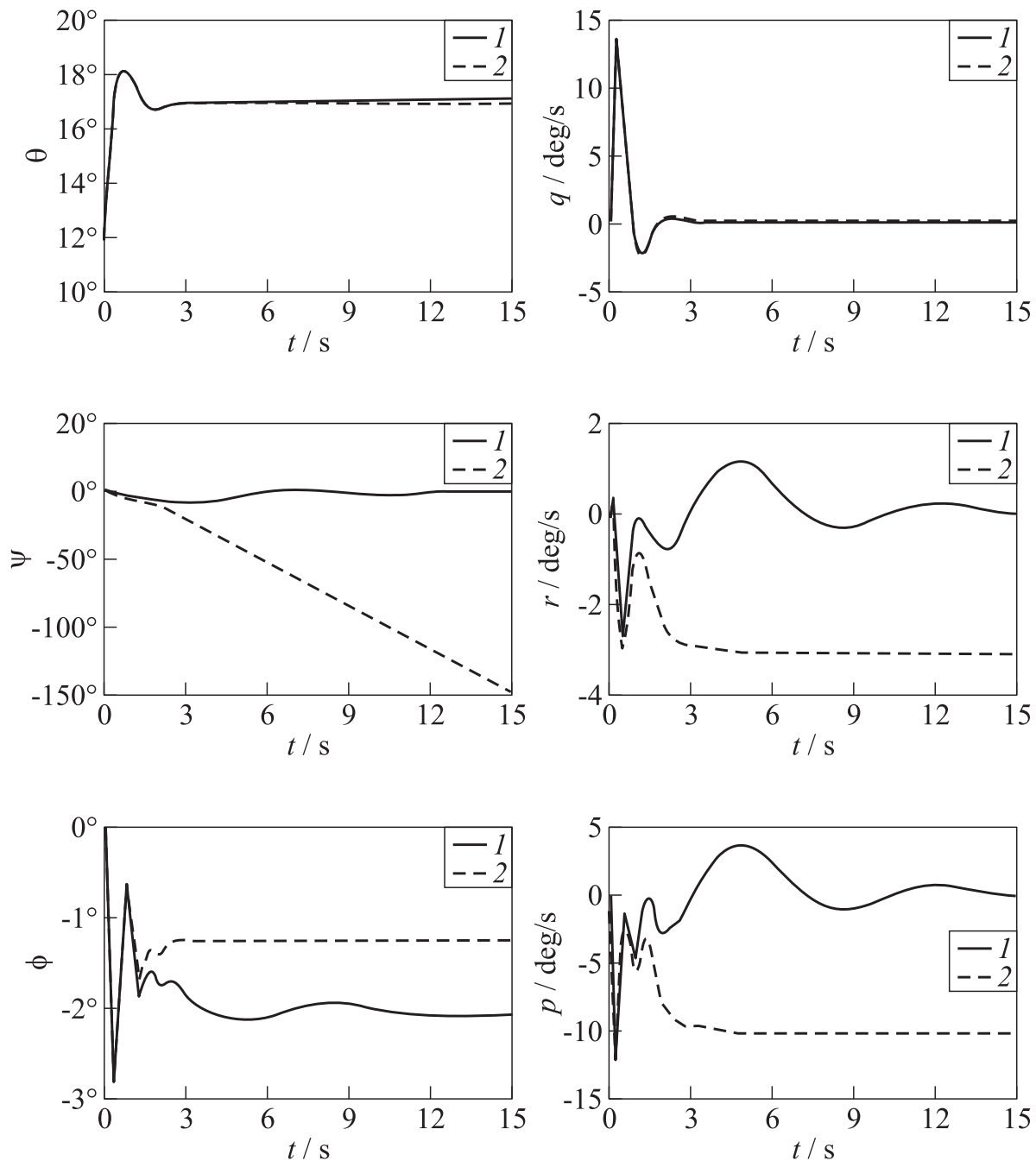

Figure 2 Aircraft dynamics for $\delta_{e}=-14^{\circ}: 1-\mathrm{NN}$ control; and $2-$ open-loop 
moments due to friction and gravitational forces are not considered). Angles of attack $\alpha$ and sideslip $\beta$ are connected with the Euler angles by the following relationships:

$$
\tan \alpha=\tan \theta \cos \phi ; \quad \sin \beta=\sin \theta \sin \phi .
$$

The comparative simulation of wing rock motion of the same dynamically scaled model in the 3DoF gimbals in the wind tunnel and in the free flight [9]
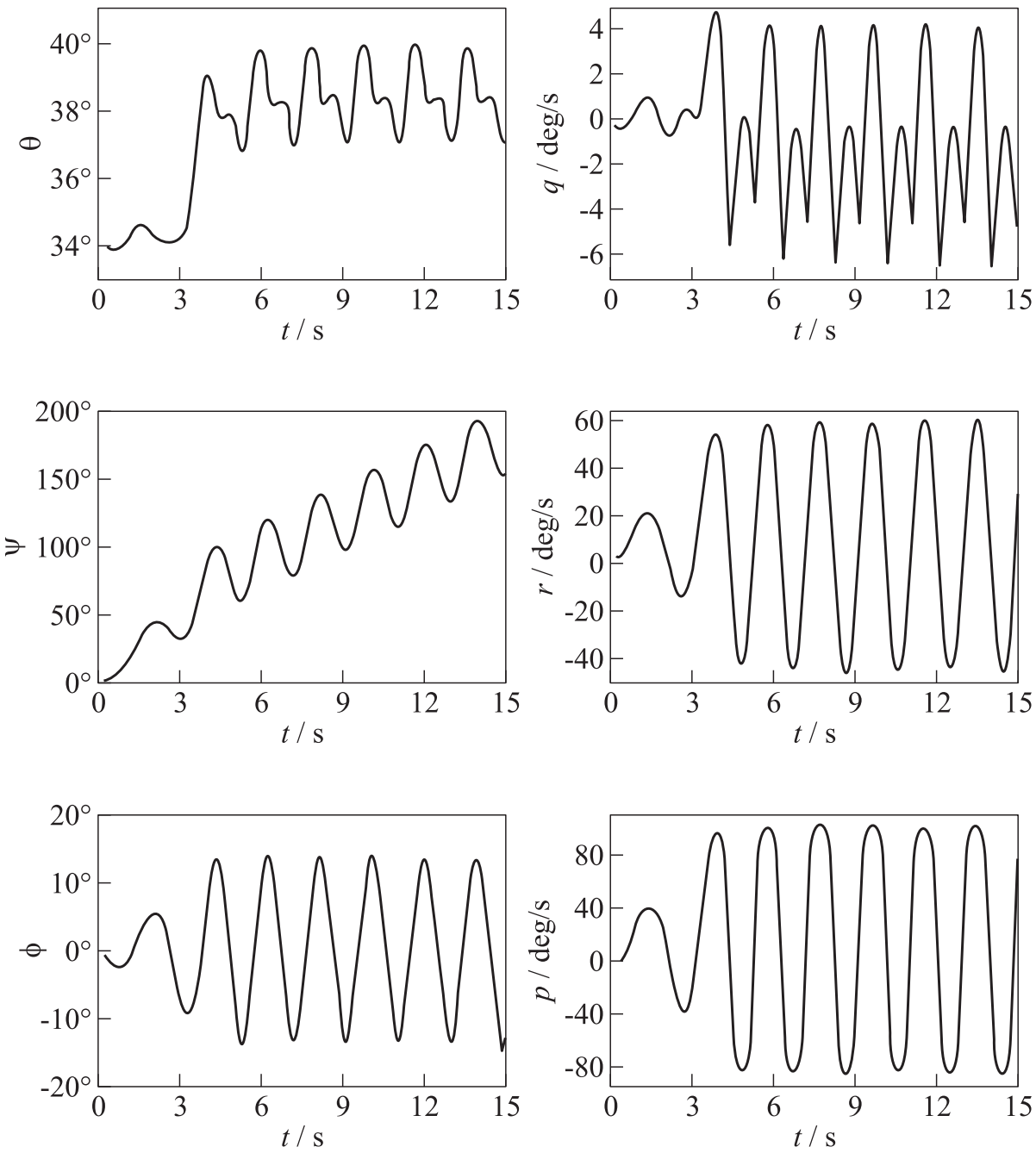

Figure 3 Dynamic of the open-loop system for $\delta_{e}=-23^{\circ}$ 
shows that amplitudes of the self-induced oscillations for the angle-of-attack and the sideslip angles are quite similar. The quantitative difference between the roll oscillation amplitudes is due to a difference in dynamic equations.

An aerodynamic model of the maneuverable aircraft [14] was used to study the aircraft model dynamics on the $3 \mathrm{DoF}$ gimbals.

Simulations of the open-loop dynamics on the 3DoF gimbals are demonstrated in Fig. 2 with the dashed lines for the elevator deflection $\delta_{e}=-14^{\circ}$. One can see that this elevator deflection corresponds to moderate angle of attack $\left(\alpha \approx 17^{\circ}\right)$ where the model is stable in roll and pitch but it has small yaw rate leading to rotation of the model. The yaw instability can be explained with the presence of small aerodynamic asymmetry term in the mathematical model of the aircraft. The closed loop behavior ("NN control") is represented on the same figure by the solid line for comparison purposes. The design of the NN control law guaranteeing stability of the system will be discussed below.

Additionally, there is a region of angles of attack $33^{\circ}<\alpha<46^{\circ}$ (angles of elevator deflection $-23^{\circ} \leq \delta_{e} \leq-27^{\circ}$ ) where the model becomes unstable in pitch and roll and the self-induced oscillations of large amplitude are observed (Fig. 3). In this region, a reconfiguration of the flow over the upper surfaces of the aircraft with separations and vortex breakdowns is observed. One can see that the elevator deflection $\delta_{e}=-23^{\circ}$ leads to nose-up motion and high-angleof-attack departure. At this elevator deflection, the motion which is similar to oscillatory spin is observed. Namely, there is the model stalling with further rotational motion with respect to the supporting sting accompanied with the oscillations in pitch and roll.

\section{ADAPTIVE CONTROL}

In this section, the control law stabilizing the model in the entire range of the elevator deflection will be discussed. The standard Model Reference Adaptive Control (MRAC) $[5,8,15]$ is used.

The dynamics of the aircraft model on the 3DoF gimbals is nonlinear and governed by Eqs. (1) and (2). A reference model for the desired response is given by the linear time invariant differential equation:

$$
\dot{\mathbf{x}}_{m}=\mathbf{A}_{m} \mathbf{x}_{m}
$$

where $\mathbf{x}_{m}(t) \in R^{n}$ is the reference state vector, while $\mathbf{x}(t) \in R^{n}$ is the state vector, and $\mathbf{A}_{m} \in R^{n \times m}$ is Hurwitz. The error is the following:

$$
\mathbf{e}=\left(e_{1}, \ldots, e_{n}\right)^{\mathrm{T}}, \quad e_{i}=x_{m i}-x_{i} .
$$


The error dynamics in the system can be considered as

$$
\dot{\mathbf{e}}=\mathbf{A}_{m} \mathbf{e}+\mathbf{B}\left[\mathbf{u}_{\mathrm{ad}}+\Delta(\mathbf{x})\right]
$$

where $\Delta(\mathbf{x})$ is an unknown dynamics of the system and $\mathbf{B}$ is the known matrix of control effectiveness.

The problem is to derive the adaptive control law $\mathbf{u}_{\text {ad }}$ matching the uncertainty $\Delta(\mathbf{x})$.

The adaptive control law is specified by the following equation:

$$
\mathbf{u}=\tilde{\mathbf{W}}^{\mathrm{T}}(t) \boldsymbol{\psi}(\mathbf{x}(t))
$$

where $\boldsymbol{\psi}: R^{n} \rightarrow R^{s}$ is the vector of basis functions $\boldsymbol{\psi}=\left[\psi_{1}(\mathbf{x}) \psi_{2}(\mathbf{x}) \cdots \psi_{s}(\mathbf{x})\right]^{\mathrm{T}}$ $\in R^{s}$ and $\tilde{\mathbf{W}} \in R^{s \times m}$ is the matrix of NN weights.

In the present study, two forms of the weight update laws are considered for comparison $[16,17]$ :

$$
\begin{aligned}
\dot{\tilde{\mathbf{W}}} & =-\Gamma \mathbf{e}^{\mathrm{T}} \mathbf{P} \mathbf{B} \boldsymbol{\beta}(x)-\sigma \tilde{\mathbf{W}}(t) ; \\
\dot{\tilde{\mathbf{W}}} & =-\Gamma \mathbf{e}^{\mathrm{T}} \mathbf{P B} \boldsymbol{\beta}(x)-\sigma\|\mathbf{e}\| \tilde{\mathbf{W}}(t)
\end{aligned}
$$

where $\Gamma>0$ is the adaptation gain and $\mathbf{P} \in R^{n \times n}$ is the positive-define solution of the Lyapunov equation [18]:

$$
\mathbf{A}_{m}^{\mathrm{T}} \mathbf{P}+\mathbf{P} \mathbf{A}_{m}=-\mathbf{Q}, \quad \mathbf{Q}=\mathbf{Q}^{\mathrm{T}}>0 .
$$

The nonlinear parameterizations of the adaptive control law via introducing the modification terms $\sigma \tilde{\mathbf{W}}(t)$ or $\sigma\|\mathbf{e}\| \tilde{\mathbf{W}}(t)$, where $\sigma>0$, are used to improve the stability $[16,17]$. The proof of stability using the Lyapunov function can be found in [5].

The control law (3) can be represented as the RBF NN, which configuration is shown in Fig. 4. The NN can be considered as a directed graph with neurons placed in it nodes. Neuron is an elementary calculating unit. The state vector $x_{1}, \ldots, x_{n}$ is the input to the $\mathrm{NN} ; \psi_{1}, \ldots, \psi_{n}$ are the activation functions of the neurons; $w_{i j}$,

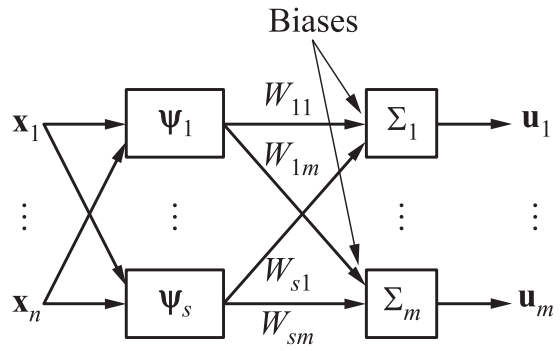

Figure 4 The RBF NN $i=1, \ldots, s, j=1, \ldots, m$, are the NN weights; $\Sigma_{1}, \ldots, \Sigma_{m}$ are the output neurons; and $u_{1}, \ldots, u_{n}$ are the NN output signals.

The main advantage of the RBF NNs is that they have the linear-in-theparameter form, as opposed to multilayer perceptron NN [19]. This enables 
quite simple NN training rules to be applied. Radial basis functions $\psi_{i}(x)$ are selected as the activation functions of the neurons. The RBF are the Gaussian functions:

$$
\psi_{i}(x)=\exp \left(-\frac{\left\|x-c_{i}\right\|^{2}}{\mu_{i}^{2}}\right), \quad i=1, \ldots, n,
$$

where $\|\cdot\|$ denotes the Euclidean norm; and $c_{i}$ and $\mu_{i}>0$ are the center and the width of the $i$ th kernel unit, respectively.

\section{SIMULATION RESULTS}

The considered system is composed of equations of motion on $3 \mathrm{DoF}$ gimbals (1) and (2), control law (3), and adaptation laws (4) or (5).

In the present study, the NN control is used both for the longitudinal and the lateral-directional control, namely, the state vector is $\mathbf{x}=(\theta, \psi, \phi, q, r, p)^{\mathrm{T}}$. A reference model for the desired response is specified by the following matrix:

$$
\mathbf{A}_{m}=\left[\begin{array}{cccccc}
0 & 0 & 0 & 1 & 0 & 0 \\
0 & 0 & 0 & 0 & 1 & 0 \\
0 & 0 & 0 & 0 & 0 & 1 \\
-\omega_{1 n}^{2} & 0 & 0 & -2 \zeta_{1} \omega_{1 n} & 0 & 0 \\
0 & -\omega_{2 n}^{2} & 0 & 0 & -2 \zeta_{2} \omega_{2 n} & 0 \\
0 & 0 & -\omega_{3 n}^{2} & 0 & 0 & -2 \zeta_{3} \omega_{3 n}
\end{array}\right]
$$

A control signal is updated discretely with time step $d t=0.02 \mathrm{~s}$ that corresponds to the real time of the wind tunnel model. Servo dynamics and position and rate limits are included in the model.

It is assumed that the servos have their own dynamics, namely, their transfer function is the following function of the first order $T=1 /(0.011 s+1)$. An additional transportation lag $(0.02 \mathrm{~s})$ is introduced. The resulting transfer function has the following form:

$$
T=\frac{\exp (-0.02 s)}{0.011 s+1} .
$$

The rates are limited to $250 \mathrm{deg} / \mathrm{s}$. The following limits are included in the model, namely, $-23^{\circ}<\delta_{e}<10^{\circ},-45^{\circ}<\delta_{\Delta e}<45^{\circ},-20^{\circ}<\delta_{a}<20^{\circ}$, and $-25^{\circ}<\delta_{r}<25^{\circ}$.

One-dimensional basis functions are used $\psi_{k i}=\exp \left(-\left(x_{k}-c_{k i}\right)^{2} / \mu_{k}^{2}\right)$ where each set $\psi_{k i}, i=1, \ldots, n$, of the functions corresponds to each state vector component $x_{k}$. The RBF centers $\mu_{k i}$ are spaced at constant interval between $x_{k_{\max }}$ 
and $x_{k_{\min }}$. For each component, the number of centers is $n=21$ and the number of $\mathrm{RBF}$ is $n \times 6=126$, correspondingly.

To achieve an appropriate overlap between neighboring kernels, their widths are adjusted according to the following rule [8]:

$$
\mu_{k}=\frac{x_{k_{\max }}-x_{k_{\min }}}{2(\sqrt{s}-1)}
$$

where $x_{k_{\max }}$ and $x_{k_{\min }}$ are the maximum and the minimum of input domain; and $s$ is the number of hidden layer neurons. The matrix from Eq. (6) is $\mathbf{Q}=\mathbf{I}$. The same parameters of the laws (4) and (5) are chosen, namely, $\Gamma=32, \sigma=1$, and the matrix of control effectiveness is the following:

$$
\mathbf{B}=\left[\begin{array}{cccc}
0 & 0 & 0 & 0 \\
0 & 0 & 0 & 0 \\
0 & 0 & 0 & 0 \\
0 & 0.1 & 0 & 0 \\
0 & 0 & 0.1 & 0.2 \\
0.01 & 0 & 0 & 0
\end{array}\right]
$$

The dynamics of the system with the adaptive control law (4) for $\delta_{e}$ $=-14^{\circ}$ is compared with the open-loop dynamics in Fig. 2. In contrast to the open-loop system, the RBF NN based control ensures yaw-stability of the model.

Simulations of the closed-loop system for $\delta_{e}=-23^{\circ}$ that corresponds to the loss of stability with different initial conditions demonstrate that the proposed control law (4) suppresses self-induced oscillations and prevent stall (Figs. 5 and 6 ). Shown in Fig. 5 are the response of the system on different initial values of $\psi=5^{\circ}$ and $10^{\circ}$. The simulations for initial values of $\phi=5^{\circ}$ and $10^{\circ}$ are shown in Fig. 6. It is seen that the controller stabilizes the wind tunnel model in both cases. In the initial period, during first $4 \mathrm{~s}, \mathrm{NN}$ is learning unknown dynamics, and after it stabilizes the system.

A comparison of the adaptation laws (4) and (5) has been carried out (Fig. 7). One can see that ' $\sigma$-modification' (4) stabilizes the system; otherwise, the 'e-modification' (5) stabilizes the system in $7 \mathrm{~s}$, but then, due to the weights grow, the system becomes unstable.

Another type of experiment has been simulated, namely, a step-wise deflection of the stabilizer from $\delta_{e}=-21^{\circ}$ up to $-31^{\circ}$. The system response is shown in Fig. 8.

Such stepwise deflection of elevator leads to variation of the angle-of-attack that covers the regions before stall $\left(\delta_{e}>-21^{\circ}\right)$, developing stall $\left(-23^{\circ} \leq \delta_{e}\right.$ $\left.\leq-27^{\circ}\right)$, and the developed stall $\left(\delta_{e}<-27^{\circ}\right)$. 



Figure 5 Neural network control for $\delta_{e}=-23^{\circ}$, different initial conditions: $1-$ $\psi=10^{\circ} ;$ and $2-\psi=5^{\circ}$

After each stepwise deflection of the elevator, one can see the decaying oscillations connected with the NN training. But the control ensures the stability of the system within the whole domain of the angles of attack.

As a result, the same RBF NN configuration with the same coefficients catches the system dynamics in the wide range of angles of attack. 

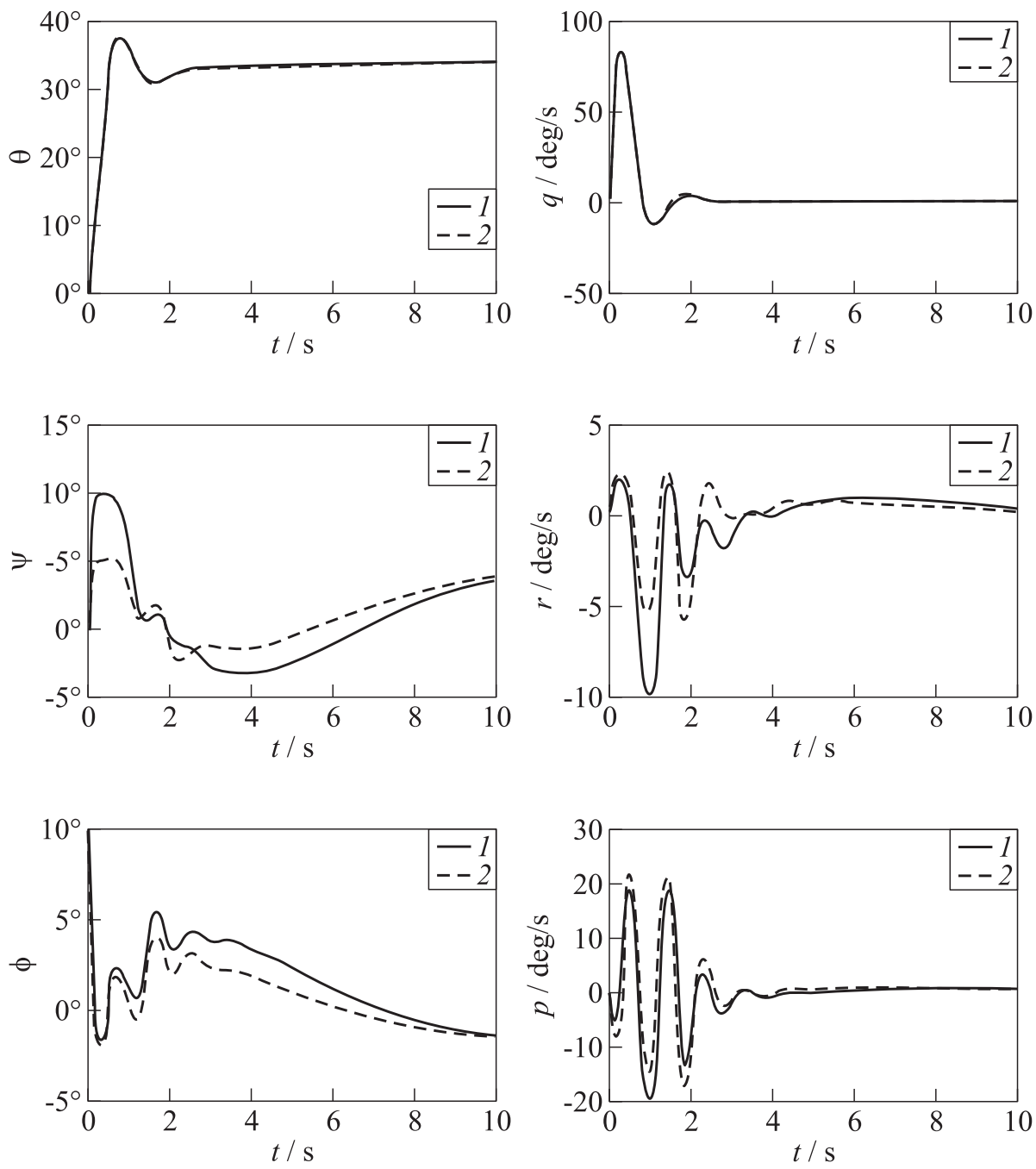

Figure 6 Neural network control for $\delta_{e}=-23^{\circ}$, different initial conditions: $1-$ $\phi=10^{\circ} ;$ and $2-\phi=5^{\circ}$

\section{CONCLUDING REMARKS}

A dynamics of the wind tunnel aircraft model in the 3DoF gimbals is examined. In the simulation, the mathematical model of the maneuverable aircraft aerodynamics is considered. The elevator, differential stabilator, ailerons, and the rudders are included in the model. The analysis is carried out both for the moderate 

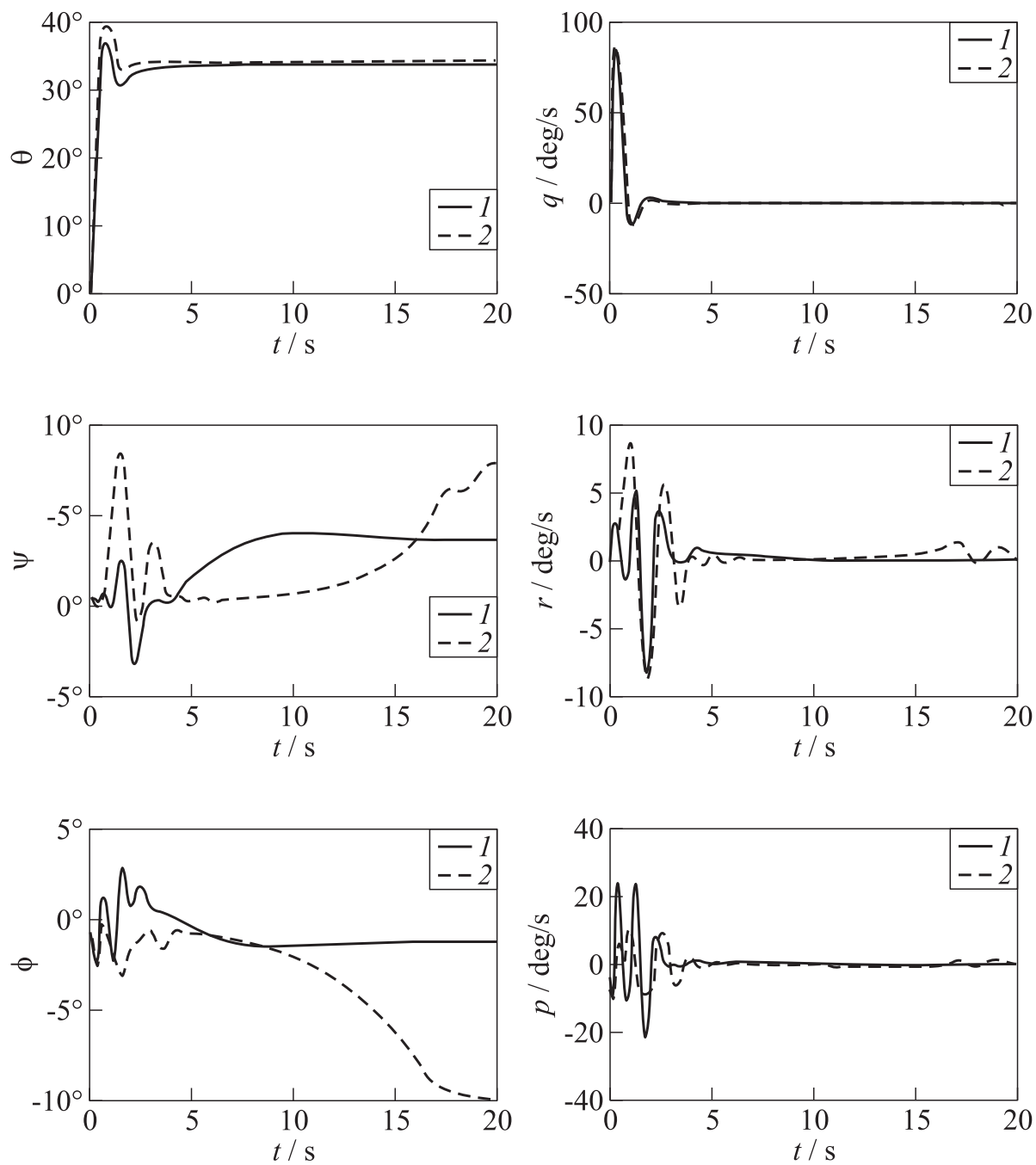

Figure 7 Comparison of nonlinear parameterization techniques, $\delta_{e}=-23^{\circ}: 1-$ $e$-modification; and $2-\sigma$-modification

and high angles of attack. At the moderate angles of attack, the model is stable in roll and pitch but has small instability in yaw. At the high angles of attack, the model exhibits unstable dynamics with tendency to stall, spin, and wing rock.

The NN adaptive control based on the MRAC technique is proposed for stabilization of the wind tunnel aircraft model in the 3DoF manoeuvre rig. For the present control design, it is considered that the pilot or other autopilot signals do 

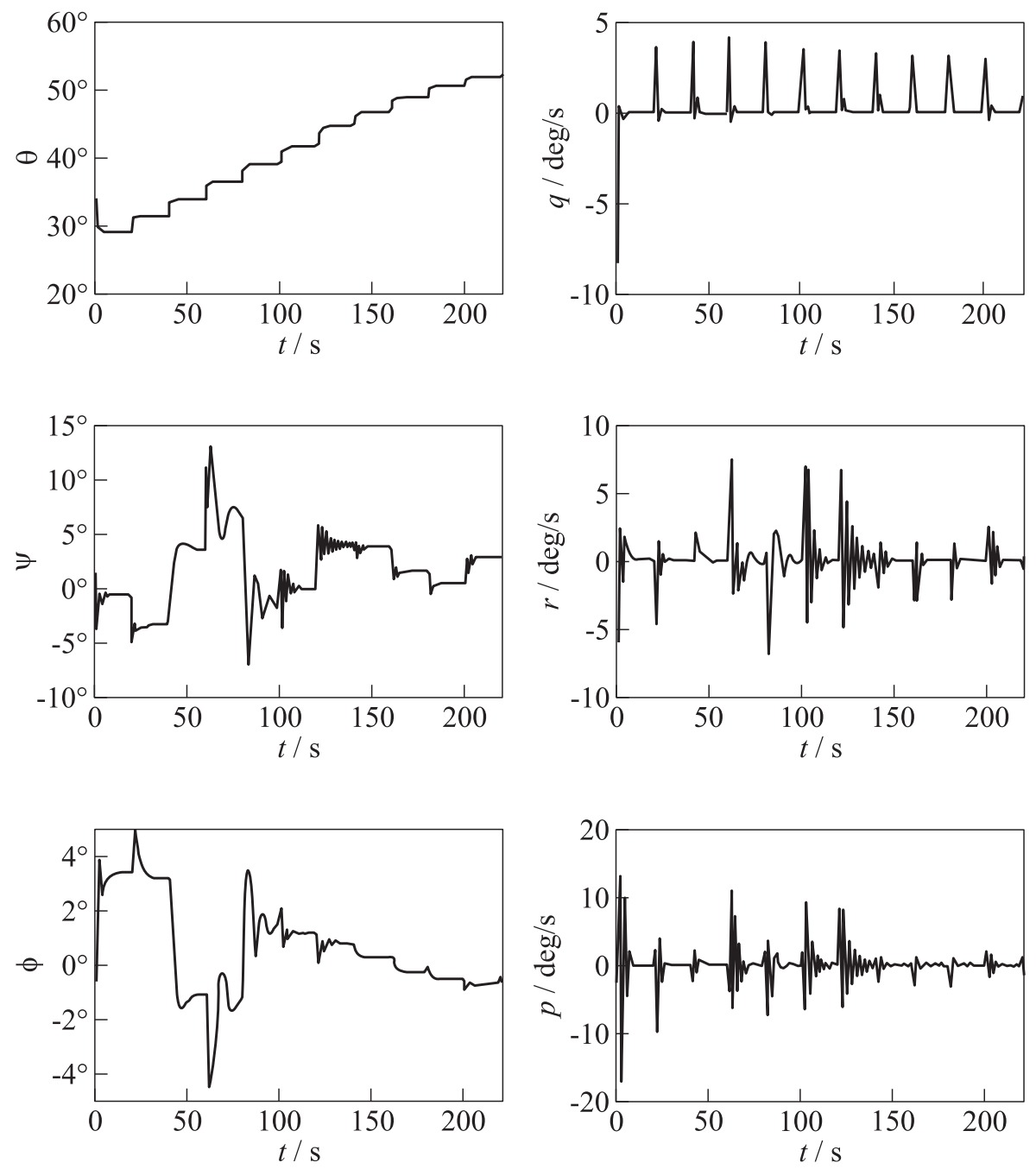

Figure 8 Neural network control for $\delta_{e}=-21^{\circ} \ldots-31^{\circ}$

not interact with the NN control while transient period in which the NN learning the unknown dynamics. Two techniques of the nonlinear parameterization of the control law, namely, ' $\sigma$-modification' and 'e-modification,' are studied and the performance of the ' $\sigma$-modification' is shown to be superior. For the control law with ' $\sigma$-modification,' different initial conditions are tested. It is shown that the considered control law ensures stability of the system from moderate up to high angles of attack, preventing wing rock, stall, and spin. 


\section{REFERENCES}

1. Schmidt, L.V. 1979. Wing rock due to aerodynamic hysteresis. J. Aircraft 16(3):129-133.

2. Hsu, C.-H., and C.E. Lan. 1984. Theory of wing rock. J. Aircraft 22(10):920-924.

3. Elzebda, J. M., A.H. Nayfeh, and D. T. Mook. 1989. Development of an analytical model of wing rock for slender delta wings. J. Aircraft 26(8):737-743.

4. Luo, J., and C. E. Lan. 1993. Control of wing-rock motion of slender delta wings. J. Guid. Control Dynam. 16(2):225-231.

5. Singh, S. N., W. Yirn, and W. R. Wellsa. 1995. Direct adaptive and neural control of wing-rock motion of slender delta wings. J. Guid. Control Dynam. 18(1):25-30.

6. Shue, S.-P., and R.K. Agarwa. 2000. Nonlinear $H_{\infty}$ method for control of wing rock. J. Guid. Control Dynam. 23(1):60-68.

7. Go, T. H., and R. Ramnath. 2002. Analysis of the two degree of freedom wing rock in advanced aircraft. J. Guid. Control Dynam. 25(2):324-333.

8. Calise, J., Y. Shin, and M. D. Johnson. 2004. A comparison study of classical and neural network based adaptive control of wing rock. AIAA Paper No. 2004-5320.

9. Khrabrov, A. N., M. E. Sidoryuk, E. N. Kolesnikov, Yu. A. Vinogradov, I. I. Grishin, and K. A. Kolinko. 2014. On possibility of critical flight regime study in wind tunnels using a three-degree-of-freedom gimbals. TsAGI Science J. 45(8):825-839.

10. Sidoryuk, M.E. 2014. Robust control design to suppress wing rock motion of a wind-tunnel aircraft model in 3DoF gimbals. TsAGI Science J. 45(8):977-992.

11. Faller, W. E., S. J. Schreck, and H. E. Helin. 1995. Real-time model of three dimensional dynamic reattachment using neural networks. J. Aircraft 32(6):1177-1182.

12. Ignatyev, D. I., and A. N. Khrabrov. 2015. Neural network modeling of unsteady aerodynamic characteristics at high angles of attack. Aerosp. Sci. Technol. 41:106115.

13. Kolmogorov, A. T. 1963. On the representation of continuous functions of many variables by superposition of continuous functions of one variable and addition. American Mathematical Society Translations 28(2):55-59.

14. Golikov, V.I., A. A. Orlov, P. N. Vlasov, and V. A. Syrovatskii. 2010. Control of lateral oscillations of maneuverable aircraft at high angles of attack. Tekhnika vozdushnogo flota LXXXIV(2(699)):17-41.

15. Aström, K., and B. Wittenmark. 1995. Adaptive control. 2nd ed. Readings: Prentice Hall. 574 p.

16. Ioannou, P., and P. Kokotovic. 1984. Instability analysis and improvement of robustness of adaptive control. Automatica 20(5):583-594.

17. Narendra, K., and A. Annaswamy. 1987. A new adaptive law for robust adaptation without persistent excitation. IEEE Trans. Automat. Contr. 32(2):134-145.

18. Gantmacher, F. R. 1959. Ch. 11: Complex symmetric, skew-symmetric, and orthogonal matrics. The theory of matrices. New York, NY: Chelsea. Vol. 2.

19. Suykens, J. A., J. P. Vandewalle, and B. L. D. Moor. 1996. Artificial neural networks for modelling and control of non-linear systems. Springer. $247 \mathrm{p}$. 